\title{
Inquiry into the potential value of an information pamphlet on consent to surgery to improve surgeon-patient communication
}

\author{
P Meredith, C Wood
}

\begin{abstract}
Objectives-To find out how patients recently undergoing surgery experienced the consenting process and the response of these patients to a pamphlet on consent to surgery. To test the reaction of health professionals to the pamphlet.

Design-A pilot pamphlet was produced and a questionnaire was sent to patients inquiring about their consenting experience, and how the pamphlet might have helped them through the consent procedure. A pamphlet and a questionnaire were also sent to a random sample of the health professionals serving these patients.

Subjects-Patients and health professionals.

Results-61\% of patients returned the questionnaire. Knowledge about the consent procedure was shown to be limited. $49 \%$ were unaware that they had the right to insist that the surgeon could only perform the specified operation and nothing more. $83 \%$ were unaware that they could add something in writing to the consent form before signing. $28 \%$ of health professionals returned their questionnaire, most of whom thought that the pamphlet provided a useful contribution to surgeon-patient communication

Conclusion-Evidence shows that patients are not well informed about consenting to surgery and further information would provide much needed guidance on understanding their role in the consent procedure. The low response from the health professional study is perhaps an indication that at present this is an issue which is not seen as a priority.
\end{abstract}

(Quality in Health Care 1998;7:65-69)

Keywords: consent; communication; pamphlet

\section{Introduction}

Traditionally within the National Health Service, consent to surgery has been managed in a highly informal way based on trust and dependency. This is a system which has worked as a convenience both for less communicative doctors and undemanding patients. It has also been found that the function and status of the hospital consent form is not entirely clear to patients nor to the doctors with the responsibility to obtain it. ${ }^{1}$ Also, research has shown how the present training of junior doctors in some surgical wards teaches them to bluff their way through the form signing ceremony. "By discouraging patients' questions they manage to hide their own ignorance, to avoid calling upon busy doctors, and to process patients smoothly through surgery towards discharge."

Patient expectations of care are increasingly influenced by the consumer standards they have come to expect in the wider commercial environment. Dissatisfaction is now made more explicit with patients increasingly willing to resort to litigation. ${ }^{3}$ This is particularly the case in the realm of consent to surgery. ${ }^{4-6}$ The problems facing patients and health professionals in securing informed consent in hospitals have been considered from several viewpoints including changing the form and the way it is administered. $^{7}$

In 1996 the Royal College of Surgeons of England designed a pamphlet on consent to surgery, with assistance from professional and consumer bodies. Its aim was to help educate patients about their rights and obligations regarding consent as a contribution to better relations between surgeons and patients in the face of rising patient expectations. It is generally thought that properly designed literature to educate patients holds great potential to improve communication and patient satisfaction and hence quality of care.

To test the design and merits of a pamphlet on this subject two surveys were conducted, one among patients and a second among health professionals. The surveys provided the opportunity to discover how patients currently experience giving their consent in the NHS and what impact the provision of information about consent may have on the doctor-patient relationship. This paper describes the findings.

\section{Methods}

SELECTION OF PATIENTS

Three hospital sites were chosen, which served inner city, suburban, and rural populations in different geographical locations (one in the south east, one in the north, and one in the south west of England). Surgeons involved in the study organised distribution from within their own hospital. The study began in March 1996 and was completed in August 1996.

The person in the hospital responsible for selection was given a target sample of 100 patients who had recently had surgical treatment and had been discharged within the past month. It was hypothesised that consent would be of differing importance according to the severity of the patient's condition, therefore the sample aimed to be divided equally between day case patients $(50 \%$ of these to have 
Introduction to surgical consent

Surgery is performed not simply to save life but to alleviate symptoms. This almost always involves a trade off of benefits against risks or side effects about which only the patient can decide.

\section{The outpatient visit}

The purpose of the meeting and the importance of consent at this stage.

A checklist of questions, the answers of which engender valid consent.

\section{The consent form}

A form is illustrated in the pamphlet and the sections elaborated and explained with illustrations.

The meaning of the signature and how the patient can use the form for their own needs. Consenting on behalf of relatives and children.

Informed consent for participation in training or clinical trials.

Box 1 Outline of pamphlet contents

undergone an endoscopy) and inpatients (30\% of these to have undergone surgery for removal of a malignancy). Selection was based on discharge and theatre records.

THE PAMPHLET

The pamphlet aimed to prepare the patient by providing examples of common questions about treatment on which informed consent depends. It also examined the components of a typical surgical consent form. Box 1 shows an outline of the pamphlet.

THE PATIENTS' QUESTIONNAIRE

A patients' questionnaire was created containing 27 questions to assess the patients' reaction to the booklet in the light of their recent consenting experience and to seek what were thought to be the strengths and weaknesses of what they had read (box 2). Both tick boxes and space for free text responses were included within the questionnaire.

Patients were posted their questionnaire directly from the hospital together with a pamphlet and an introductory letter explaining the survey. Those who did not return their questionnaire within three weeks were sent a reminder letter. Another reminder and copy of the questionnaire and pamphlet were sent after a further three weeks if there was still no response.

THE HEALTH PROFESSIONALS' QUESTIONNAIRE This questionnaire contained only open ended questions. The main theme of the questions was the perceived effect of the pamphlet's widespread availability on doctor-patient interaction and the efficiency of the consultation process (box 3). Each hospital provided the Royal College of Surgeons of England with a list of all the surgical nursing staff, surgeons, and general practitioners serving the hospital. All surgeons and a random sample of the nurs-
(Q1) How did you feel about the decision which led to your operation?

I felt the surgeon took the time to discuss the options for treatment and involved me fully in the decision.

I felt the decision was made of my behalf which I went along with.

(Q 2) What in your opinion is the most important signal a patient can give that they are consenting to an operation?

Agreeing to what the surgeon proposes in the first outpatient meeting

Coming into hospital

Signing the consent form

No signal is more important than another

(Q3) How much attention did you give your consent form?

A lot of attention

Some attention

Not much attention

(Q4) How easy did you find your consent form to understand?

Very easy Quite easy Not easy

(Q5) How much importance did you attach to signing your consent form?

I felt it was very important

I felt it was quite important

I did not think it was important

I can't remember

(Q6) Were you aware that you had the right to insist that the surgeon may only perform the operation specified and nothing more?

Yes No

(Q7) Before reading this pamphlet, did you know that a patient could state in writing on the form what they did not want a surgeon to do during their operation?

Yes No

(Q8) Before reading this pamphlet, did you know that it may not necessarily be a patient's consultant (or the surgeon who would carry out the operation)

Yes No

(Q9) Are there any sections of the pamphlet which could have been easier to understand?

Yes No

(Q10) What difference, if any, has the pamphlet made to your understanding about consenting to surgery? (open ended)

(Q11) What is the most important point raised by the pamphlet? (open ended)

(Q12) Who do you think would find this pamphlet useful?

All patients

Only some patients with certain conditions No patients

Box 2 A selection of the contents of the patients' questionnaire

ing staff and general practitioners (due to the large number) were posted a questionnaire, explanatory letter, and a pamphlet. No reminder letters were sent.

\section{DATA ANALYSIS}

Questionnaire data were analysed with SPSSwin software. $\chi^{2}$ Tests were conducted when appropriate, to which all $\mathrm{p}$ values relate. All free text replies were categorised according to the 
Table 1 Sample distribution by category

\begin{tabular}{lcc}
\hline Category & $\begin{array}{c}\text { Questionnaires distributed } \\
(n(\%))\end{array}$ & $\begin{array}{l}\text { Questionnaires returned } \\
(n(\%))\end{array}$ \\
\hline Day patient, endoscopy & $30(11)$ & $17(57)$ \\
Day patient, other procedure & $45(17)$ & $22(49)$ \\
Inpatient, malignant & $60(22)$ & $37(62)$ \\
Inpatient, other & $135(50)$ & $88(65)$ \\
Total & $270(100)$ & $164(61)$ \\
\hline
\end{tabular}

Table 2 Age of respondents

\begin{tabular}{lc}
\hline Age range & $n(\%)$ \\
\hline $18-24$ & $3(2)$ \\
$25-34$ & $18(11)$ \\
$35-44$ & $29(19)$ \\
$45-54$ & $25(16)$ \\
$55-64$ & $33(21)$ \\
$65-74$ & $34(22)$ \\
$75-84$ & $13(8)$ \\
$\geqslant 85$ & $2(1)$ \\
Total & $157(100)$
\end{tabular}

Seven missing cases. common positive and negative themes emerging from the comments. Quotations aim to be representative of these themes.

Results

THE PATIENT SURVEY

Response rates

A total of 270 questionnaires were posted to patients. Only one hospital did not complete their distribution by the closing date due to other audit activities delaying the start of the selection. This hospital distributed 70 questionnaires. Of the 270 distributed questionnaires, 164 were returned giving a response rate of $61 \%$ (55\% including non-distributed questionnaires). The final sample distribution by category was $10 \%$ day case endoscopy, $13 \%$ day case other, $23 \%$ inpatient cancer, and 54\% inpatient other (table 1).

The median age range of patients was 55-64 (table 2 ). Of the day cases $62 \%$ were $<55$ years of age compared with $43 \%$ of the inpatients $\left(\chi^{2}=4.01 ; \mathrm{df}=-1, \mathrm{p}<0.04\right)$. A question sought to identify any respondents whose first language was not English, but there were few cases and no significant conclusions could be drawn.

THE PATIENTS' QUESTIONNAIRE

Experience of consenting to surgery

(Q1) Decision making-Asked how much patients thought they were involved in deciding about their operation 100/153 (65\%) patients thought that their surgeon had fully involved them. Notably, over one third of the respondents $(53(35 \%))$ thought that the decision was made on their behalf, which they merely went along with. This may be explained by some patients' free text comments in which they drew a distinction between trivial and serious surgery, usually for the purpose of placing themselves in the former category-for example, day surgery; endoscopies. However, there was no significant variation between responses of day patients and inpatients $\left(\chi^{2}=0.002: \mathrm{df}=1\right.$, $\mathrm{p}>0.9)$.

(Q2) Indications of consent-Respondents were asked what they thought was the most important signal they can give the doctor that they are consenting to a procedure-for example by verbally concurring with their surgeon on treatment; signing the consent form, or entering hospital. The results show no common agreement about the status of the consent form (table 3). Forty three per cent thought that agreeing with the surgeon at the outpatient clinic was the most important signal, with $32 \%$ stating that the signing of the consent form was the most important signal. Day patients were overrepresented in this group $\left(\chi^{2}=15.51: \mathrm{df}=3\right.$, $\mathrm{p}<0.005)$.
(Q3) Attention given to consent form-41/148 $(28 \%)$ patients thought that they gave the form a lot of attention. Sixty five (44\%) patients thought that they gave it some attention, and $42(28 \%)$ thought that they did not give it much attention. There was no statistical indication that those patients who underwent an operation for cancer paid any more attention to the form than the others $\left(\chi^{2}=11.50: \mathrm{df}=6, \mathrm{p}>0.05\right)$.

Day patients paid less attention to the form than inpatients $\left(\chi^{2}=6.37: \mathrm{df}=2, \mathrm{p}<0.04\right)$.

(Q4) Understanding the consent form-Over one third of the respondents, 49/139 (35\%) found the consent form very easy to understand, most (84 (60\%)) found it quite easy, and only six $(5 \%)$ found it difficult. Some patients commented that their ability to comprehend the form was compromised by the manner in which it was presented for signature. Thus several respondents thought that they had had insufficient time to absorb the contents of the form or felt pressured because a doctor stood waiting for them to sign it immediately before their operation.

(Q5) Importance of signing the form-Asked how much importance they attached to signing their consent form, just over half (78/150 $(52 \%))$ thought that it was very important with a further $48(32 \%)$ thinking that it was quite important. Only 16 respondents (11\%) thought that it was not important. Eight $(5 \%)$ could not remember.

(Q6, Q7) Additions to the consent formPatients were asked if they knew that they had the right to insist that the surgeon could only perform the specified operation and nothing more, and $77 / 152(51 \%)$ patients stated they knew this to be so, leaving 75 (49\%) who did not. Only a few, 25/148 (17\%) knew that they could express these wishes in writing on the consent form.

(Q8) Operating surgeon-Patients were asked if they knew that a surgeon other than their consultant may carry out their operation; $98 / 153(64 \%)$ stated that they knew this.

\section{Design and impact of the pamphlet}

(Q9, Q10) Understanding of the pamphlet$139 / 141(99 \%)$ patients reported that in their view nothing in the pamphlet could have been made easier to understand. A free text box provided the opportunity for the respondents to explain if and how the pamphlet had made any difference to their understanding about consent. Forty patients added something in their own words. The following examples are representative of these comments:

"I did not realise that I had as much to say about what happened as I obviously have."

"It has made me aware that I am my own person and can decide what happens to me."

"It has made it more clear to me that I have the right to be properly informed; that I have the right to ask questions."

(Q11) Most important aspect of the pamphletAsked what they thought was the most 
Table 3 The important signals of consent

\begin{tabular}{lc}
\hline Response & $n(\%)$ \\
\hline $\begin{array}{l}\text { Agreeing to what the surgeon proposes at } \\
\text { the outpatient meeting }\end{array}$ & $68(43)$ \\
Signing the consent form & $50(32)$ \\
Coming into hospital & $20(13)$ \\
No signal is more important than another & $18(11)$ \\
Other & $5(1)$ \\
Total & $161(100)$ \\
\hline
\end{tabular}

Three missing cases.

important part of the pamphlet, in the light of their experience, the largest proportion of those responding cited the ability to write something themselves. Other mixed comments are indicative of the realisation that the form is designed to serve the patient as much as the hospital:

"I could read what the surgeon writes on the consent form, and could state on the form what I do not want the surgeon to do."

"I am the most important person rather than the consultant or doctor."

“The limitations on the surgeons' rights because of the patients' rights."

"Side effects are risks (to be taken into consideration)."

"Being able to understand the consent form."

"The pamphlet has made me aware of the questions I should have asked even though I was only going for day surgery."

(Q12) Benefit of the pamphlet-Asked who the patient thought would benefit from the pamphlet, 134/146 (92\%) thought that all patients would benefit. Ten $(7 \%)$ thought that some patients - those undergoing more risky, major surgery - would need such a pamphlet. Only two (1\%) thought that the pamphlet was not useful to any patient.

THE HEALTH PROFESSIONALS' QUESTIONNAIRE A pamphlet and questionnaire were posted to 404 health professionals, and 115 were returned, giving an overall response rate of $28 \%$. Of 74 surgeons contacted, 26 responded (35\%), of 123 surgical nursing staff, 27 responded $(22 \%)$, and 62 out of 207 general practitioners responded $(30 \%)$. The results are based on responses from open ended questions relating to the idea of providing such information to patients (box 3).

Of those responding, nearly all the surgeons $(25 / 27(95.5 \%))$ and general practitioners $(60 / 62(97 \%))$ thought that the idea of a pamphlet was an excellent or good idea. The following statements show some reasons for their support:

Surgeons

"Make communication between surgeon and patient easier and more effective."

"It should enhance communication."

"It will encourage patients to think and ask questions which may not otherwise have come
(Q1) What do you feel about the idea of providing patients with a pamphlet on consent to surgery? (open ended)

(Q2) What effect do you think the pamphlet may have on the existing communication patients have with surgeons and other health professionals? (open ended)

Box 3 The questions to the health professionals

up, and knowing this will encourage surgeons to communicate better."

Nursing staff

"Will provide a basis for doctors to start talking to their patients."

General practitioners

"It will help patients make a valid decision."

"It will concentrate the mind on issues that might cause concern."

"Consultants will be encouraged to improve consent procedures rather than patients coming to general practitioners for further explanations."

"It may involve more time explaining things to the patient, needing a trial to see if this is the case."

Some respondents acknowledged that the consent procedure is not always treated with the time and importance it deserved:

Surgeons

"Having read the pamphlet I would probably go through the consent form more fully."

\section{Nursing staff}

"It is of major concern to nurses that informed consent is sometimes denied in a fast moving environment."

"It should make it easier for patients to ask for information, as not all (junior) staff give appropriate information."

"It will reduce inhibitions of patient as many surgeons are inaccessible gods on pedestals."

Some respondents, although in support of the idea of providing information, thought that conditional factors should be taken into account:

\section{Surgeons}

"It may involve more time explaining things to the patient, needing a trial to see if this is the case."

Nursing staff

"Good idea but only if used together with proper time for patient to ask questions."

"The pamphlet must not be allowed to be the only form of communication."

"Most patients feel very detached from their surgeon; this may make them seem even more removed and be an excuse for the surgeon to take less time with them." 
General practitioners

"It clarifies what patients can expect of surgery. However, it may unrealistically raise expectations of the level of explanation leading to longer appointments."

"It must not be a substitute for personal communication."

"This is the responsibility of the surgeon, rather than a pamphlet."

"This will bounce back on general practitioners, increasing their work load. Also patients will think that they have to take more responsibility for their treatment and become confused."

\section{Discussion}

This study sought to explore how patients experienced consenting to surgery and how the provision of an information pamphlet explaining the consent procedure would help guide and improve the involvement of patients in this process.

Our evidence showed that patients are not well informed about consenting to surgery and that further information would provide much needed guidance on understanding their role in the consent procedure. The low response from the health professionals is perhaps an indication that at present this is an issue which is not seen as a priority.

There were several limitations to this study. One is that the study was retrospective, asking patients who had already had an operation how they may have benefited from such information being made available rather than looking at an active process of consent. Another limitation was that the use of a postal questionnaire is by its nature limited in the amount of in depth information that can be gained. However, despite this the data do provide a positive indication of the need for more guidance and information on the rights of patients.

Results show that there were gaps in patients' knowledge of the consent procedure-for example, $49 \%$ were unaware that they could specify that the surgeon could only undertake the operation specified. Results also show the benefit that such a pamphlet could have on the communication process, with most patients (92\%) thinking that all patients could benefit from such information being made available. Research has shown that whenever patients are given the opportunity to request information, they do so. ${ }^{8-10}$ This also seems to hold true for information about the consent procedure.

The response from health professionals was disappointingly low, and the opportunity should have been taken to send reminder letters, although it is uncertain how much this may have improved the response rate. The low response can perhaps be seen as an indication of the lack of importance given to the consent procedure by health professionals. It is interesting to note that although only $28 \%$ of patients gave the consent form much attention, 52\% thought that signing the consent form was very important. This response may be influenced by the often rushed manner that the form is presented to patients with little time or encouragement provided to study the form in detail.

However, the comments received from the health professionals were encouraging and although it cannot be assumed that these comments are representative of all those posted a questionnaire, it does show a level of support for use of a patients' pamphlet to improve the current communication between doctors and patients.

Many health professionals are aware of the limitations of the consent form, which is seen by many as unproductive and confusing. It was not the objective of this study to consider alternatives to the consent procedure but to look at the current situation for patients undergoing surgery and to create useful guidance information to help with this process.

Patient information literature plays a vital part in informing patients and is an important component of the quality of care provided by the National Health Service. The subject of consent has long been neglected in provision of information and this study shows that such literature can provide a much needed tool to improve communication and understanding between patients and the health professionals that serve them.

We thank the staff of the hospitals organising and taking part in this study, and the Royal College of Surgeons of England for financial support.

1 Richardson N, Jones P, Thomas M. Should house officers obtain consent for operation and anaesthesia. Health Trends 1996;28:56-9.

2 Alderson P. Consent to surgery. Hospital Update 1992; July: 529-31.

3 Audit Commission What seem to be the matter? Communication between hospitals and patients. London: HMSO, 1993.

4 Tribe DMR, Korgaonkar GJA. Liability and litigation trends. Br F Hosp Med 1990;43:217-20.

5 Chatterson $v$ Gerson and another. London: Medical Law Review Cases, 1992; 80

6 Rogers $v$ Whitaker. London: Medical Law Review Cases, 1994; 148.

7 Taylor B. Time to question forms of consent [editorial]. Hospital Doctor 1996;61:17-8.

8 Wallace L. Informed consent to elective surgery: the therapeutic value? Soc Sci Med 1986;22:29-33.

9 Meredith P. Patient participation in decision making and consent to treatment: the case of general surgery. Sociology Health and Illness 1993;15:115-36.

10 Williams O. Patient knowledge of operative care. $7 \mathrm{R}$ Soc Med 1993;86:328-31. 